\title{
Timing of Kocher maneuver in laparoscopic endoscopic cooperative surgery for duodenum tumor: Before or after endoscopic submucosal dissection?
}
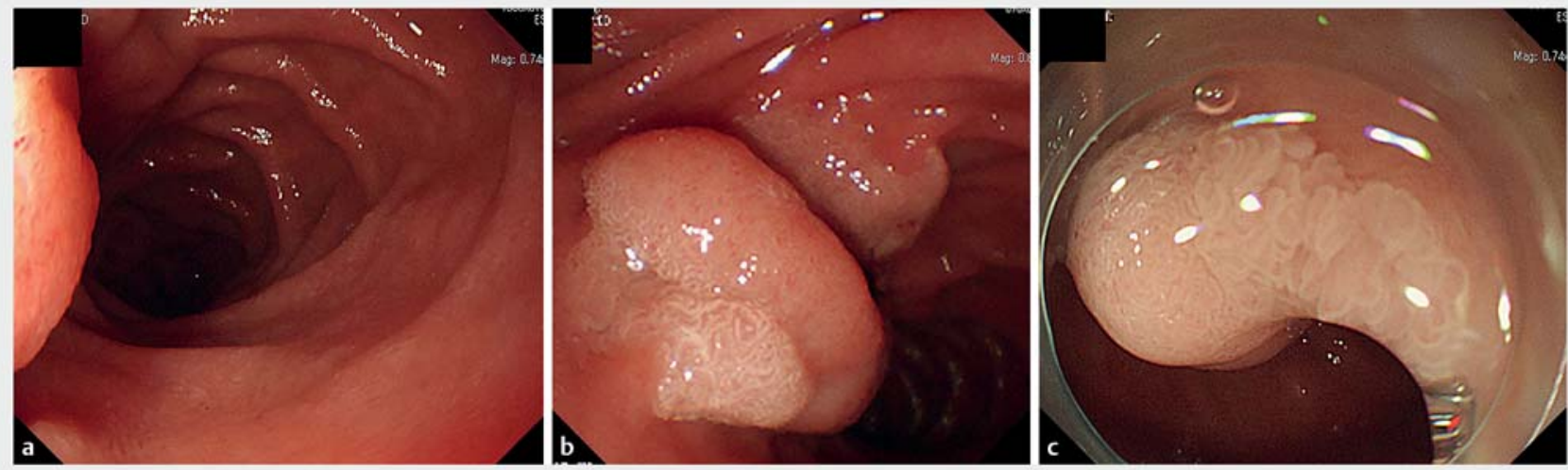

- Fig. 1 a Preoperative forward-viewing endoscopy did not provide adequate visualization due to unstable access to the duodenal tumor b Only side-viewing endoscopy provided a view sufficient for evaluation. c Intraoperative endoscopic view of the duodenal tumor with a forward-viewing endoscope after Kocher maneuver, showing an image entirely different from that obtained before the maneuver.

We read with great interest the recent article by Otowa and colleagues [1]. They retrospectively evaluated the short-term outcomes of 10 patients with superficial non-ampullary duodenal epithelial tumors (SNADET) treated by laparoscopic endoscopic cooperative surgery for duodenum tumor (D-LECS) and reported their techniques in detail. Based on the high rate of intraoperative perforation in their study $(40 \%)$, ensuring confirmation and reinforcement of the thin duodenal wall appears to be mandatory after endoscopic submucosal dissection (ESD). Given that duodenal ESD alone is associated with a high rate of complications, such as delayed perforation [2], we totally agree with them that laparoscopic seromuscular suture would effectively prevent such an event.

D-LECS consists mainly of ESD and laparoscopic reinforcement. The former is essential for this procedure. Because loss of duodenal fixation on the retroperitoneum caused difficulty with ESD, Otowa and colleagues clearly advocated the Kocher maneuver, mobilization of the duodenum and head of the pancreas from the retroperitoneum, not being completed before ESD. However, whether this procedure is optimally performed before versus after ESD depends on duodenal tumor location.

A 56-year-old man underwent screening esophagogastroduodenoscopy, which revealed a villous protruding lesion located slightly distal to the superior duodenal flexure. Forward-viewing endoscopy did not provide acceptable tumor visualization ( Fig.1a), necessitating the use of a side-viewing endoscope for precise examination ( $>$ Fig. 1b). The pathological diagnosis, based on the biopsy specimen, was tubular adenoma. Accordingly, the ESD technique did not seem to be technically feasible due to unstable access to the duodenal tumor using a forward-viewing endoscope, such that D-LECS would be contraindicated. We used the upfront Kocher maneuver to fully detach the duodenum from the retroperitoneum, to modify accessibility to the tumor with a forward-viewing endoscope (FVE). As expected, the endoscopic appearance on the FVE was effectively changed ( $\mathbf{F i g . 1 c}$ ) and an adequate visual field was obtained during ESD. After completion of ESD, the sero-

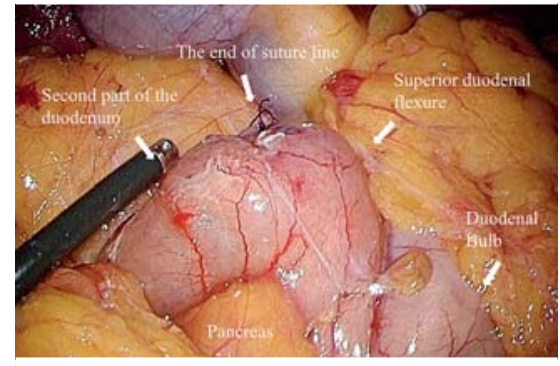

- Fig. 2 Intraoperative findings after laparoscopic seromuscular suture at the ESD site, located at the dorsal portion of the beginning of the second part of the duodenum.

muscular layer at the ESD site was reinforced laparoscopically ( $>$ Fig. 2).

Increasing duodenal mobility by applying the Kocher maneuver might hamper the duodenal ESD procedure [1]. However, our case clearly raises the possibility that altering duodenal anatomy is worth trying to facilitate endoscopic treatment of tumors previously assumed to be unsuitable for this procedure by endoscopists. Because D-LECS appears to be the optimal treatment option for SNADET, collaborative trials involving 
surgeons and endoscopists are encouraged to establish and promote safe management of this tumor.

Competing interests

$\mathrm{KH}$ have received lecture fees from KARL STORZ Endoscopy Japan and Ethicon. TG have received lecture fees from FUJIFILM Medical, FUJIFILM, Olympus, MC Medical, Daiichi Sankyo, AstraZeneca, EA Pharma, AbbVie GK, Mitsubishi Tanabe Pharma, Boston Scientific and Viatris. HY have received lecture fees from Taiho Pharmaceutical, Ono Pharmaceutical, Bristol Myers Squibb, Viatris, Otsuka Pharmaceutical Factory, Kaken Pharmaceutical, Daiichi Sankyo, Eli Lilly and Ethicon.

The authors

\section{Ken Hagiwara ${ }^{1}$, Ryoji Ichijima² ${ }^{2}$ Takuji}

\section{Gotoda $^{2}$, Hiroharu Yamashita ${ }^{1}$}

1 Department of Digestive Surgery, Nihon University School of Medicine, Tokyo, Japan

2 Division of Gastroenterology and Hepatology, Department of Medicine, Nihon University School of Medicine, Tokyo, Japan
Corresponding author

\section{Hiroharu Yamashita MD, PhD}

Department of Digestive Surgery, Nihon University School of Medicine, 1-6 KandaSurugadai, Chiyoda-ku, Tokyo, 101-8309, Japan

Fax: +81-3-3292-2880

hyamashi-tky@umin.net

\section{References}

[1] Otowa Y, Kanaji S, Morita Y et al. Safe management of laparoscopic endoscopic cooperative surgery for superficial nonampullary duodenal epithelial tumors. Endosc Int Open 2017; 5: E1153-E1158

[2] Marques ], Baldaque-Silva F, Pereira P et al. Endoscopic mucosal resection and endoscopic submucosal dissection in the treatment of sporadic nonampullary duodenal adenomatous polyps. World J Gastrointest Endosc 2015; 7: 720-727

\section{Bibliography}

Endosc Int Open 2022; 10: E224-E225

DOI 10.1055/a-1723-3516

ISSN 2364-3722

(c) 2022. The Author(s).

This is an open access article published by Thieme under the terms of the Creative Commons Attribution-NonDerivativeNonCommercial License, permitting copying and reproduction so long as the original work is given appropriate credit. Contents may not be used for commercial purposes, or adapted, remixed, transformed or built upon. (https:// creativecommons.org/licenses/by-nc-nd/4.0/)

Georg Thieme Verlag KG, Rüdigerstraße 14, 70469 Stuttgart, Germany

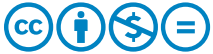

